\title{
Thermal components in the early X-ray afterglow of GRBs
}

\author{
D. Lazzati ${ }^{\star}$ \\ Institute of Astronomy, University of Cambridge, Madingley Road, Cambridge CB3 OHA, UK \\ Received 15 October 2002 / Accepted 11 December 2002

\begin{abstract}
The presence of thermal components in the early X-ray afterglows of $\gamma$-ray bursts is investigated. We discuss both the presence of a thermal continuum and, in particular, of collisional X-ray emission lines. We compute the predicted luminosity by a thin plasma for a range of metallicities for the continuum and the $\mathrm{K}_{\alpha}$ lines of the elements $\mathrm{Mg}, \mathrm{Si}, \mathrm{S}, \mathrm{Ar}, \mathrm{Ca}$ and $\mathrm{Fe}$. We show that light travel effects are dominant in the determination of the thermal continuum and line luminosities, and derive the relevant equations. We conclude that thermal lines and continua are unlikely to dominate the early afterglow of GRBs, unless the explosion site is surrounded by a very massive and extremely clumped shell of material. Such conditions are difficult to envisage in the close environment of the GRB progenitor, unless they are excited by some strong precursor activity, like in the Supranova scenario.
\end{abstract}

Key words. gamma-rays: burst - line: formation - radiation mechanisms: thermal

\section{Introduction}

Almost all the $\gamma$-ray bursts (GRBs) detected so far are associated with a transient X-ray afterglow (Lazzati et al. 2002a). This afterglow is supposed to be due to the early deceleration of the fireball by the interstellar medium (Meszaros \& Rees 1997) and its radiation produced by non-thermal synchrotron. Such an interpretation is corroborated by spectral (van Paradijs et al. 2002) and polarimetric (Covino et al. 1999) observations.

More recently, several X-ray afterglows observed by Newton-XMM had a spectrum that can be better fit by an optically thin thermal bremsstrahlung model (Watson et al. 2002) rather than with an absorbed power-law (even though the latter model cannot be unambiguously ruled out on purely statistical grounds). In particular, several high ionization emission lines were detected in the early afterglow of GRB 011211 (Reeves et al. 2002, hereafter R02) which can be accounted for, together with the observed continuum, by a moderately enriched thermal plasma.

The possibility of a thermal origin of the X-ray lines detected in several afterglows was first discussed in Lazzati et al. (1999; see also Vietri et al. 1999; Kumar \& Narayan 2002), who pointed out how a 10 times solar enriched plasma may produce a $\mathrm{Fe} \mathrm{K}_{\alpha}$ line with the observed large luminosity and equivalent width. In this paper we derive more rigorously the conditions for the environment and the heating mechanism that must be satisfied in order to observe line and continuum emission with the prescribed luminosities, equivalent widths (EWs) and variability time scales.

\section{Continuum and line emission in thermal plasma}

To compute the continuum emission from a thermal optically thin plasma we adopt the treatment and basic equations of Rybicki \& Lightman (1979). To compute the luminosities of $\mathrm{K}_{\alpha}$ lines from a thermal plasma, instead, we adopt the widely used code MEKAL (Mewe et al. 1985; Liedahl et al. 1995) as implemented in XSPEC (Arnaud 1996). We concentrate in particular the elements $\mathrm{Mg}, \mathrm{Si}, \mathrm{S}, \mathrm{Ar}, \mathrm{Ca}$ and $\mathrm{Fe}$, for which emission lines have been detected in the afterglow of GRBs. In Fig. 1 we show the line production efficiency for an optically thin thermal plasma as a function of the plasma temperature and for several values of metallicity. We plot $\eta_{\text {line }}$, i.e. the ratio of the $\mathrm{K}_{\alpha}$ emission line for the six elements above, irrespective of their ionization state, over the total luminosity of the plasma. Gray shading highlights regions in which the equivalent width (EW) of the lines is less than $100 \mathrm{eV}$, a robust lower limit to any emission feature detected in the afterglow so far.

When using the MEKAL code to evaluate line emission luminosities, one has to remember that the code does not include any radiation transfer, since it assumes that the medium is optically thin to radiation. The actual optical depth of a cloud of plasma at temperature $T$ depends on the temperature and on the frequency of the radiation considered. When X-ray continuum radiation is considered, the optically thin approximation can be used up to column densities $N_{\mathrm{H}} \lesssim \sigma_{T}^{-1} \sim 1.5 \times 10^{24} \mathrm{~cm}^{-2}$. If, however, line emission is concerned, it must be taken into account that intermediate-high $Z$ elements retain some electrons which may cause the plasma to be optically thick due to photoionization. In Fig. 2 we show the optical depth of a solar metallicity plasma with $N_{\mathrm{H}}=1.5 \times 10^{24} \mathrm{~cm}^{-2}$ as a function of frequency for a range of temperatures between $10^{6}$ and $10^{8} \mathrm{~K}$.

\footnotetext{
^ e-mail: lazzati@ast.cam.ac.uk
} 


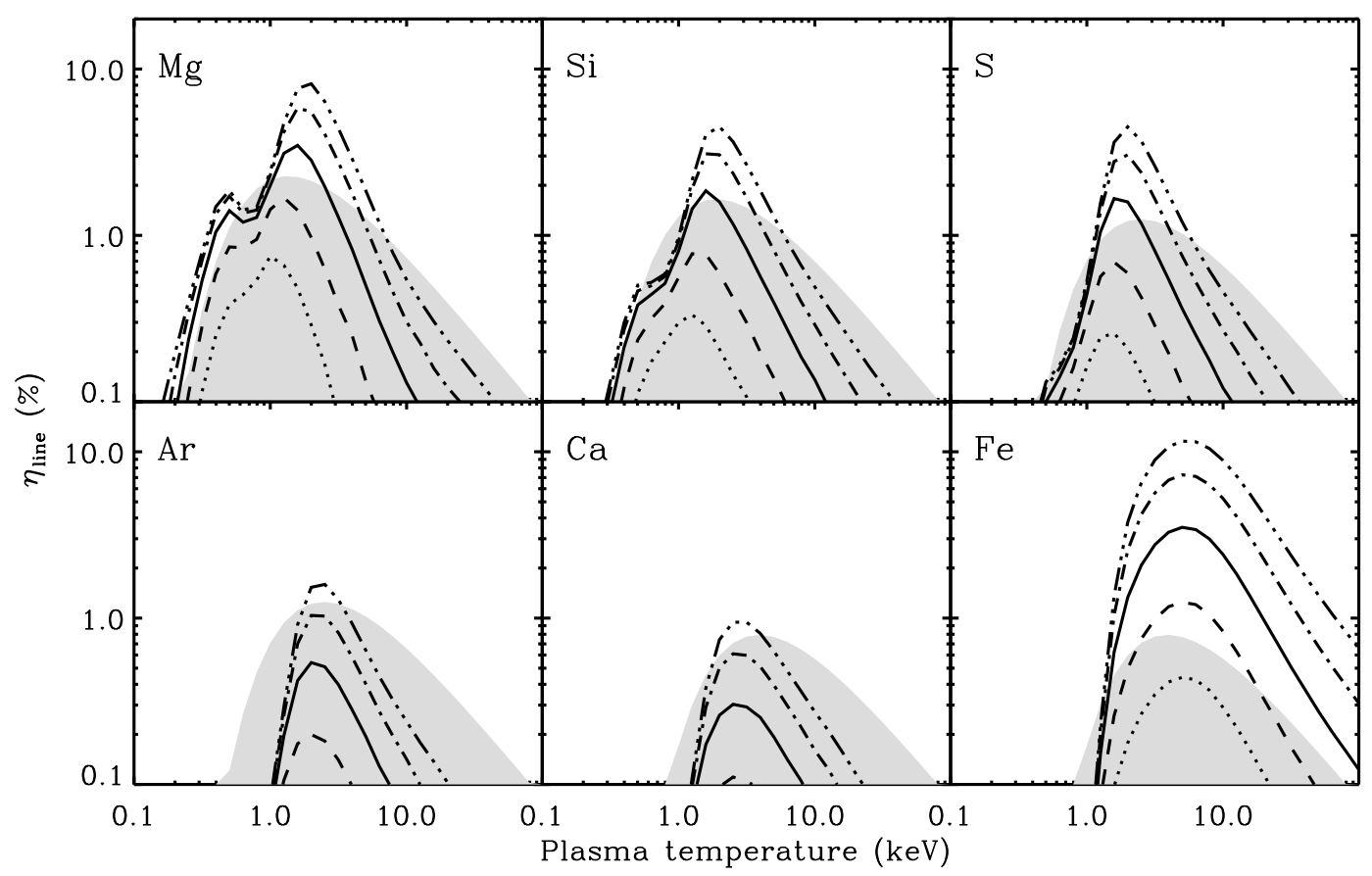

Fig. 1. Efficiency of $\mathrm{K}_{\alpha}$ line emission for several elements as a function of the temperature in an optically thin plasma. The parameter $\eta_{\text {line }}$ is defined as the line luminosity over the bolometric luminosity of the thermal plasma. Each panel shows 5 different lines. From top to bottom these refer to a metallicity $10,3,1,0.3$ and 0.1 solar. The gray shading highlights regions in the parameter space where an equivalent width lower than $100 \mathrm{eV}$ is predicted.

The opacity of a cold gas is also shown for comparison. In the region of the considered emission lines, the plasma can be optically thick up to temperatures of several keV. This will limit the maximum line luminosity and EW: increasing the column density of the plasma will have no effect on the line luminosity since line photons will be able to escape freely only out of the optically thin surface layer of the medium. Instead of properly introducing radiation transfer in the optically thin MEKAL code, which is a formidable task, in the following we will assume that, after the plasma becomes optically thick to radiation at the line frequency, the line luminosity does not increase if the column density of the gas is increased (see Lazzati et al. 2002b for discussions on a similar assumptions in reflection models).

\section{Thermal models for GRB afterglows}

Consider a cloud of plasma of electron density $n$ at a temperature $T$ which covers a solid angle $\Omega=2 \pi(1-\cos \theta)$ along the line of sight to the observer at a distance $R$ from the burst explosion site (see Fig. 3). The luminosity that is inferred from infinity, taking into account the light travel time effects, is given by ${ }^{1}$ :

$$
L \approx 1.7 \times 10^{-23} T_{8}^{1 / 2} E I \frac{t_{\text {heat }}+t_{\text {cool }}}{R / c(1-\cos \theta)+t_{\text {heat }}+t_{\text {cool }}}
$$

where $E I$ is the emission integral $\left(E I=\int_{V} n_{\mathrm{e}} n_{\mathrm{i}} \mathrm{d} V\right), t_{\text {cool }} \sim$ $1.2 \times 10^{15} T_{8}^{1 / 2} n^{-1} \mathrm{~s}$ is the plasma cooling time and $t_{\text {heat }}$ is the heating time, i.e. the time during which heat (or energy) is supplied to the emitting plasma. It is assumed that during this

\footnotetext{
${ }^{1}$ Here and in the following we indicate a quantity $Q$ as $Q=10^{x} Q_{x}$. We also adopt CGS units.
}

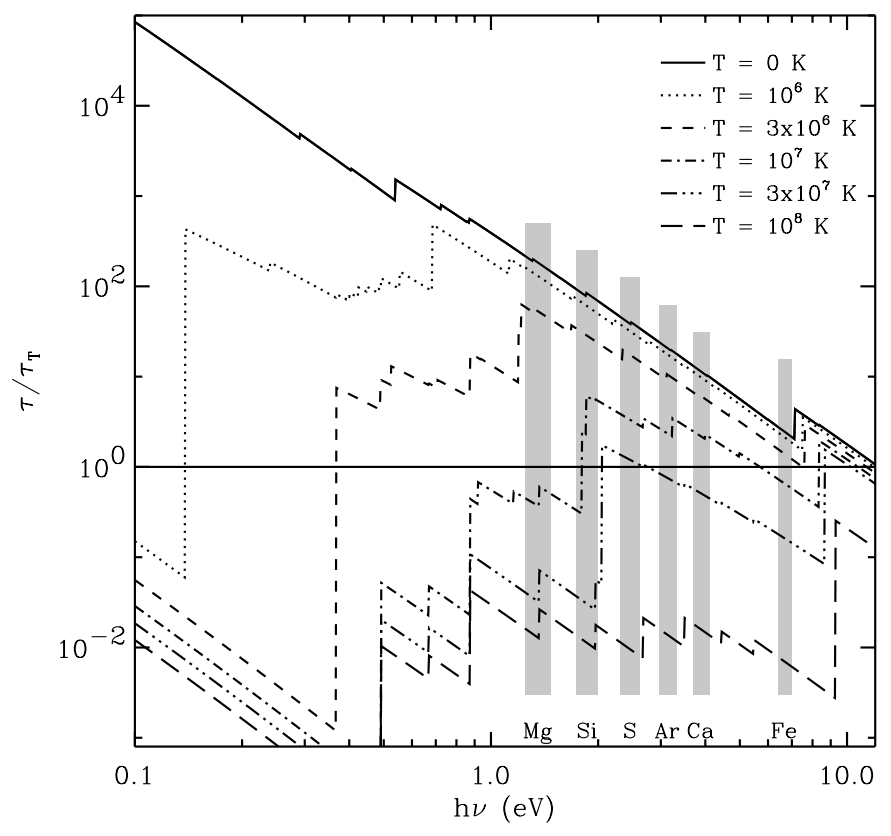

Fig. 2. Photoionization optical depth of a $\tau_{T}=1$ plasma in thermal equilibrium as a function of the temperature and frequency. The range of frequencies of the considered $\mathrm{K}_{\alpha}$ lines is shown by gray shading.

heating time the temperature is held constant, i.e. the plasma is in equilibrium. In the following we will consider a uniform density plasma and approximate $n_{\mathrm{e}} \approx n_{\mathrm{i}} \approx n$. In this case $E I=n^{2} V$, where $V$ is the volume of the emitting cloud.

In many astrophysically relevant situations (e.g. the IGM in galaxy clusters), $R / c \ll t_{\text {cool }}$ and both the heating and cooling 
time scales are irrelevant and Eq. (1) simplifies to the usual free-free equation:

$L=1.7 \times 10^{-23} T_{8}^{1 / 2} E I$.

In GRBs this may not be the case. In order to reproduce afterglow observations in the non-cooling regime, the plasma cloud must satisfy simultaneously three conditions. First, it must produce the observed luminosity in the non-cooling regime

$L=1.7 \times 10^{-23} T_{8}^{1 / 2} n^{2} \frac{4 \pi}{3} R^{3} \eta_{R} \frac{\Omega}{4 \pi} \sim 10^{46} L_{46} \quad \frac{\mathrm{erg}}{\mathrm{s}}$

where the numeric value is typical for the early X-ray afterglow (see e.g. GRB 011211, R02) and $\eta_{R}$ is the volume filling factor of a possible shell-like or clumpy cloud. Second, it must fulfill the non-cooling condition:

$R(1-\cos \theta) \leq c t_{\mathrm{cool}}=3.6 \times 10^{25} T_{8}^{1 / 2} n^{-1}$

finally, it must require a total amount of energy smaller than the total energy of a GRB:

$E=8.7 \times 10^{-8} T_{8} n R^{3} \eta_{R} \frac{\Omega}{4 \pi} \leq 10^{52} E_{52} \quad$ erg.

Conditions (3) and (4) yield the density constraint:

$n \leq 3.7 \times 10^{8} T_{8}^{2} \eta_{R} L_{46}^{-1} \frac{\Omega}{4 \pi}$

while conditions (3) and (5) give:

$n \geq 1.2 \times 10^{9} T_{8}^{1 / 2} L_{46} E_{52}^{-1}$.

The above conditions cannot clearly be satisfied simultaneously if all the parameters are taken equal to their fiducial values. Since $\eta_{R}$ and $\Omega / 4 \pi$ are both numbers smaller than unity, a change in the geometry does not help, making the constraint of Eq. (6) more stringent. Also the temperature cannot be changed significantly, especially as long as X-ray lines must be taken into account.

In conclusion, a thermal component that contributes to the early afterglow of a typical GRB cannot be emitted in the non-cooling regime. In fact, in order to reach a luminosity large enough without involving a too large thermal energy, the plasma density must be so large as to make the cooling time very short. As an example, R02 derived a cooling time of $\sim 2 \mathrm{~s}$ for the afterglow of GRB 011211. We cannot therefore adopt the simple Eq. (2) but we must use the more complete Eq. (1).

We therefore consider in the following two limiting cases: $R / c(1-\cos \theta) \gg t_{\text {cool }} \gg t_{\text {heat }}$ (flash heating) and $R / c(1-$ $\cos \theta) \gg t_{\text {heat }} \gg t_{\text {cool }}$ (steady heating). In principle also the case $t_{\text {heat }} \gg R / c(1-\cos \theta) \gg t_{\text {cool }}$ may be interesting. It is however difficult to identify a heating source that can be active for a time comparable to the line emission time scale. Even if the emitting plasma would be heated by afterglow photons, the heating time could be at most a few per cent of the line emission time scale (Lazzati et al. 2002b).

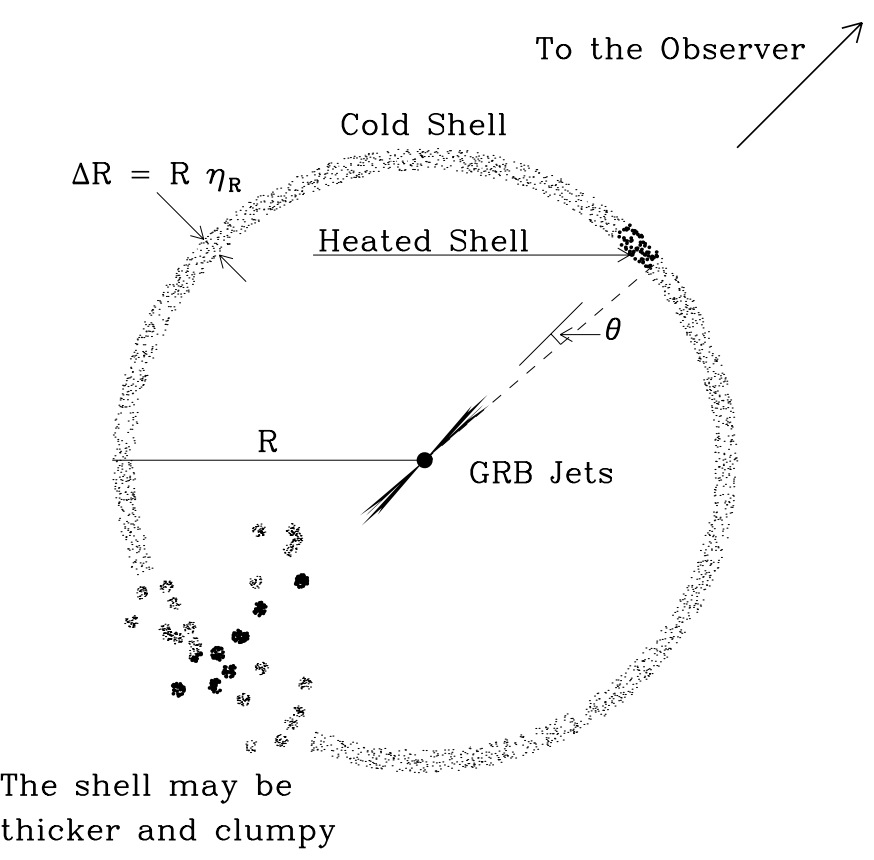

Fig. 3. Cartoon of the geometrical set-up for the GRB and thermal reprocessing material. The GRB is surrounded by a thin shell (or clumped thicker shell) of material, which is heated by GRB photons within the fireball solid angle.

\subsection{Flash heating}

Consider a portion of a spherical shell of plasma of radius $R$, temperature $T$ and uniform density $n$ covering a solid angle $\Omega=1-\cos \theta$ (see, again, Fig. 3). Its emission integral can be written as $E I=n^{2} R^{3} \eta_{R} \Omega$ where $\eta_{R} \equiv \Delta R / R$ is the ratio of the thickness of the shell to its radius ${ }^{2}$. Considering the values $\eta_{\text {line }}$ defined above (and shown in Fig. 1), the cloud will produce a line with luminosity ${ }^{3}$ (see Eq. (1)):

$L_{\text {line }}=1.9 \times 10^{27} \eta_{\text {line }} R T_{8} \tau_{T}$

where we have used the Thomson opacity $\tau_{T}=n R \eta_{R} \sigma_{T}$. Equation (8) coupled with the efficiencies shown in Fig. 1 implies that when we observe a line with luminosity $L_{\text {line }} \gtrsim 5 \times$ $10^{44} \mathrm{erg} \mathrm{s}^{-1}$ (Ghisellini et al. 2002) a thermal model in the flash heating regime requires a radius larger than $R \gtrsim 2 \times 10^{17} \mathrm{~cm}$. Analogous results are obtained by imposing a ten times larger continuum luminosity. Such a large radius implies that the line luminosity should remain constant for a time $t=R(1-\cos \theta) / c$, to be compared with the observed variability time scales $t_{\mathrm{var}} \sim$ $2 \times 10^{4}$ s observed in e.g. GRB 011211 (R02). This constraint can be satisfied if the heated plasma covers a very small solid angle, of opening angle $\theta \sim 4.5^{\circ}$, at the limit but consistent with the smallest opening angles inferred for GRB jets from afterglow modelling (Frail et al. 2001; Panaitescu \& Kumar 2002). In order to satisfy the cooling condition, however, the plasma must be dense $\left(n \gtrsim 10^{11} \mathrm{~cm}^{-3}\right)$ and therefore confined in a very

\footnotetext{
${ }^{2}$ Note that if a clumpy medium is assumed instead of a spherical shell, $\eta_{R}$ is equal to the volume filling factor of the configuration that maximizes the emitted luminosity.

3 The continuum luminosity can be easily derived by adopting $\eta_{\text {line }}=1$.
} 


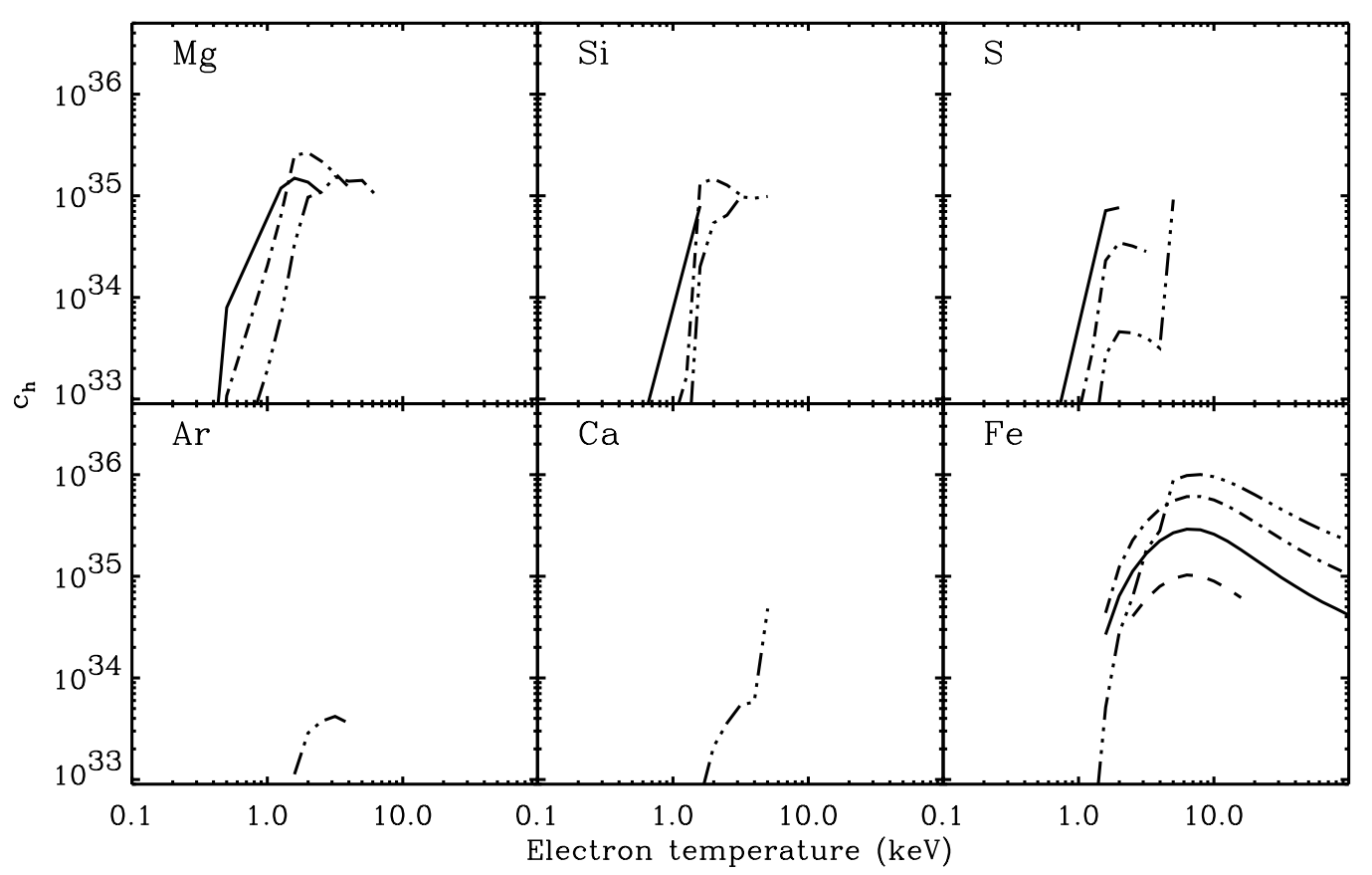

Fig. 4. The luminosity coefficient $c_{\mathrm{h}}$ (see text) as a function of the plasma temperature and of metallicity for the considered elements. As in Fig. 1, the dotted, dashed, solid, dot-dash and dot-dot-dot-dash lines are relative to $0.1,0.3,1,3$ and 10 time solar metallicity, respectively.

thin shell (or small clumps) with $\eta_{R} \lesssim 5 \times 10^{-5}$. In addition, if we assume that the plasma is due to a spherical shell surrounding the GRB explosion site which is heated only in the small polar cap by afterglow photons, the shell mass would turn out to be $M=4 \pi R^{2} m_{\mathrm{p}} \tau_{T} / \sigma_{T} \sim 600 M_{\odot}$ for the case explored above. Both the geometry factor $\eta_{R}$ and the total mass required seem to be rather extreme, even though the whole picture is, in this case, completely self consistent.

\subsection{Steady heating}

Let us now consider a plasma in which a source of heating is active for a time scale $t_{\text {heat }}>1.2 \times 10^{15} T_{8} / n=t_{\text {cool }}$. In the case of radiative heating, this time scale may be for example the GRB duration or even longer, if the afterglow photons can contribute to the heating. In steady heating, the line luminosity (continuum luminosity for $\eta_{\text {line }}=1$ ) can be written as:

$L_{\text {line }}=7.2 \times 10^{36} \tau_{T}^{2} T_{8}^{1 / 2} \eta_{\text {line }} \frac{t_{\text {heat }}}{\eta_{R}} \equiv c_{\mathrm{h}} \frac{t_{\text {heat }}}{\eta_{R}}$

where we have defined the parameter $c_{\mathrm{h}}$ in order to emphasize the ratio of the heating time scale over the geometry parameter. In Fig. 4 we show the highest possible values of the coefficient $c_{\mathrm{h}}$ as a function of temperature for five different values of metallicity (as in Fig. 1). The lines are plotted only when the equivalent width of the line is predicted to be larger than $100 \mathrm{eV}$, corresponding to the unshaded areas in Fig. 1.

To compare these results with afterglow data, consider a Fe line with $L=10^{45} \mathrm{erg} \mathrm{s}^{-1}$ (GRB 991216; Piro et al. 2000) or the $S$ line in GRB 011211 (R02) with luminosity $L=4 \times 10^{44} \mathrm{erg} \mathrm{s}^{-1}$. In both cases a ratio $t_{\text {heat }} / \eta_{R} \gtrsim 10^{9}$ is required, by comparison with the appropriate panel in Fig. 4. A typical heating time can be considered to be the fireball transit time, which is equivalent to the total duration of the prompt
GRB emission. In both cases such duration is of the order of $\sim 100 \mathrm{~s}$, leaving us with an extreme requirement of anisotropy $\eta_{R} \lesssim 10^{-7}$. Alternatively, the heating may be provided radiatively by the absorption of GRB and early afterglow photons. In that case, the duration of the heat supply may be of several per cent of the line emission time scale (Lazzati et al. 2002b). In that case the constraint would be relaxed to the (yet still challenging) value $\eta_{R} \lesssim 10^{-6}$. The most convenient solution is represented by an (unknown) heating mechanism acting for a time comparable to the line emission time scale itself. In this case, the constraint on the geometric factor $\eta_{R}$ would be similar to the one derived above for the flash heating case. Such a heating mechanism is however presently obscure and will have to face the problem of stability discussed below (Sect. 4).

In a steady heating scenario, therefore, thermal lines and continua can dominate the early afterglow emission, only in case of extreme geometric conditions, in which the emission is produced either in a sheet like shell or an extremely clumpy medium. Interestingly, analogous extreme conditions were independently inferred for the environment of GRB 000210 (Piro et al. 2002) in order to account for the lack of ionization features in the soft X-ray afterglow spectrum. Piro et al. (2002, and references therein) argue also that such conditions may be realized in giant molecular clouds. Stability considerations (see Sect. 4), however, show that more extreme conditions are required in this case. Unlike what is derived in the flash heating condition, in this case the radius of the shell does not have to be very large, and therefore the total mass required is not huge.

\section{Stability}

In the above section we have derived some geometrical constraints on the emitting plasma in order to reproduce the 
observed features and continua. Since the results envisage particular geometric conditions, we here analyze their stability. First, since the heating energy is supposed to come from a central point (the GRB progenitor), one has to make sure that the emitting material is not accelerated to high velocities.

Consider a shell of mass $M$ absorbing energy from a relativistic outflow. If the material is radiative, it acquires a bulk velocity $v=E /(M c)$. A bulk velocity $v \simeq 0.1 c$ was measured in GRB 011211 (R02) and GRB 991216 (Piro et al. 2000). Requiring that our optically thin shell is accelerated to a comparable or smaller speed implies a radius larger than:

$R \geq \frac{L \sigma_{T}}{4 \pi m_{\mathrm{p}} c^{2} \eta_{\text {line }} \tau_{T} v}=3 \times 10^{13} \frac{L_{\text {line }, 45}}{\eta_{\text {line }} \tau_{T} v_{9}} \quad \mathrm{~cm}$.

This is not a compelling limit, given the radii discussed above.

Provided that the emitting medium is not accelerated to relativistic speeds by the energy input, we also want that the thin emitting shell (or blobs) do not expand in a time scale smaller than the emission one (which can be either the heating or cooling time scale). The shell (or blobs) was in fact in equilibrium with the ambient medium when it was cold. Now that its temperature is increased it will tend to expand under the effect of the increased internal pressure. If it expands at the speed of sound $c_{\mathrm{s}}$, its density will be sizably modified in a time scale $t_{\exp }=R \eta_{R} / c_{\mathrm{s}}$. It is therefore required that the expansion time is longer than the emission time scale.

In the case of steady heating we obtain:

$R>c_{\mathrm{s}} \frac{t_{\text {heat }}}{\eta_{R}}=10^{8} T_{8}^{1 / 2} \frac{t_{\text {heat }}}{\eta_{R}} \gtrsim 10^{17} T_{8}^{1 / 2}$

Also in the steady heating case, therefore, the radius has to be large in order to allow for the production of thermal components in the early GRB X-ray afterglows. For such large radii, however, the density required to fulfill the steady heating conditions is $n \gtrsim 10^{15} / t_{\text {heat }} \gtrsim 10^{12}$, several orders of magnitudes larger than what is inferred by Piro et al. (2002). It seems therefore that the steady heating case requires more extreme conditions than the flash heating one (the same distance from the explosion site and limits on the total mass involved, but larger densities and smaller filling factors). Applying the same stability condition to a flash heating case, we are left with the more relaxed constraint

$\tau_{T}>0.1$

which is therefore not difficult to fulfill. These stability considerations suggest therefore that the only viable way to produce a sizable thermal afterglow component is by heating a small portion of a massive $\tau_{T} \sim 1$ (either very thin or clumpy) shell of material located at a relatively large distance from the burst explosion site (Fig. 3). It should however be emphasized that these stability considerations cannot be applied if the heating is provided hydrodynamically, in such a way that the source of heating is providing also the confining pressure. In this case also a steady heating scenario, with a much smaller shell, may be viable.

\section{Clumpiness of the GRB ambient medium}

The above discussion shows that the emission lines detected in several GRB afterglows can have a thermal origin only if the GRB surroundings are extremely clumpy, with density and geometric contrasts of order of a hundred thousand or more. We now discuss the reliability of these conditions in different GRB progenitor scenario.

In the hypernova scenario, the GRB sets on simultaneously to a supernova explosion, and the ambient medium is the result of the interaction of the pre-SN star with its surroundings. In particular, the nearby ambient medium will be dominated by the late stages of the mass ejection history of the star. These stages are known to be unsteady and clumps or shell-like structures can be envisaged. If the mass ejection is caused by radiative effects, however, extreme structure cannot be produced, since the stellar luminosity varies on time scales comparable to the Kelvin time-scale, which is of the order of hundreds of years, yielding a thick shell. In the wind environment of SN1998bw, for example, Li \& Chevalier (1999) found that inhomogeneities up to a factor of a few were present. A more appealing scenario, in this perspective, is the supranova model by Vietri \& Stella (1998), where the GRB is supposed to explode several weeks to years after a supernova. The supernova explosion, having a much smaller time scale, can generate a more extreme geometry. The clumpiness of SN ejecta has been investigated by numerical simulations. It is found that Rayleigh-Taylor instabilities can produce high density clumps with angular scales $\gtrsim 1^{\circ}$ (Böttcher et al. 2002). Such structures have $\eta_{R} \equiv a / R \sim 10^{-2}$ where $a$ is the clump radius, much larger than the value required to produce sizable line emission from collisional excitation. It is however possible, in the supranova scenario, that the remnant is illuminated by a super-Eddington relativistic wind (Vietri \& Stella 1998; Konigl \& Granot 2002) in the time span between the SN and GRB explosions. The interaction of this wind with the ejected $\mathrm{SN}$ shell may increase the inhomogeneities originally present in the shell (Guetta \& Granot 2003; Lazzati \& Rees, in preparation).

\section{Discussion}

In at least three XMM-Newton spectra of X-ray GRB afterglows, the presence of a thermal component has been claimed (R02; Watson et al. 2002). We have studied the geometrical conditions that the thermal material must satisfy in order to contribute significantly to the early X-ray afterglow. We first concluded that the time scale during which the emission is observed must be set by the light crossing time of the emitting region. This implies that standard free-free equations that relate the emission integral to the luminosity cannot be used and a more general equation (Eq. (1)) has to be adopted. The implications of this are of great importance. In fact, the use of the standard formalism (Eq. (2)) to compute the EI led R02 to underestimate the emission integral for GRB 011211 by a factor $\sim 10^{4}$, and conclude that thermal emission from a shell with $R \sim 10^{15} \mathrm{~cm}$ can be a self consistent solution. We showed that, with the correct treatment of light-crossing effects, this is not the case. We then studied the possibility of (i) flash heating and 
(ii) steady heating. In both cases, we conclude that the thermal material must be extremely clumped in order to contribute significantly to a typical X-ray afterglow. In the steady heating case, the thermal material is continuously heated for a time longer than the cooling one, reaching a stationary state (albeit for a time smaller than the light-crossing one). We showed that in this case the material has to be so extremely clumped that the clumps are dissolved by the increased temperature in a very short time scale, so that an additional source of confinement must be envisaged. In the case of flash heating, a self consistent solution can be found, requiring a less extreme (even if yet compelling) clumping. In this case, the thermal material must be located far from the source and therefore, in order to preserve the fast time variability, only a small portion of it has to be heated. Assuming that the same density is spread all over the GRB explosion site, we derive that the total mass involved is large $\left(\sim 600 M_{\odot}\right)$, larger than even a type-II supernova remnant. A moderate asymmetry of the remnant could however ameliorate this requirement. It is interesting to note that, even though such clumpy and dense environments seem extreme, they were already inferred independently to account for the lack of ionization features in GRB 000210 (Piro et al. 2002) and to explain the high energy emission in GRB 940217 (Katz 1994).

To date there is not yet compelling evidence that there are indeed thermal components in the early X-ray afterglow of GRBs. It is however intriguing then in three out of four afterglows observed by XMM-Newton a thermal model yields a better fit than an absorbed power-law (R02, Watson et al. 2002a). In the case of GRB 011211 (R02) it is particularly difficult to explain the absence of an iron or nickel line, while reflection models seem to give a better explanation of the observed line ratios (Lazzati et al. 2002b).

Nonetheless, should a thermal component be confirmed in the X-ray afterglow of at least a sub-fraction of GRBs, it would be difficult to avoid the conclusion that (i) the GRB explosion site is surrounded by a massive shell of matter, similar to a 1-2 year old supernova remnant (as expected in some versions of the Supranova model, Vietri \& Stella 1998); (ii) that this remnant is extremely clumped, suggesting that some precursor activity has been taking place. Whether such a clumpiness can help make the prompt emission in an external shock scenario (Dermer et al. 2000) heavily depends on the presence of clumping at smaller scales, a test that cannot be done with thermal emission from the clumps, since it would contribute in any case a negligible flux to the early afterglow emission.

Acknowledgements. I am grateful to G. Ghisellini, P. Kumar, M. J. Rees and F. Tavecchio for useful comments and discussions. I acknowledge financial support from the PPARC.

\section{References}

Arnaud, K. A. 1996, in ASP Conf. Ser., Astronomical Data Analysis Software and Systems V, ed. G. H. Jacoby, \& J. Barnes (San Francisco: ASP), 17

Berger, E., Sari, R., Frail, D. A., et al. 2000, ApJ, 545, 56

Böttcher, M., Fryer, C. L., \& Dermer, C. D. 2002, ApJ, 567, 441

Covino, S., Lazzati, D., Ghisellini, G., et al. 1999, A\&A, 348, L1

Dermer, C. D., Böttcher, M., \& Chiang, J. 2000, ApJ, 537, 255

Frail, D. A., Kulkarni, S. R., Sari, R., et al. 2001, ApJ, 562, L55

Ghisellini, G., Lazzati, D., Rossi, E., \& Rees, M. J. 2002, A\&A, 389, L33

Guetta, D., \& Granot, J. 2003, MNRAS, in press [astro-ph/0208156]

Königl, A., \& Granot, J. 2002, ApJ, 574, 134

Kumar, P., \& Narayan, R. 2002, ApJ, submitted [astro-ph/0205488]

Lazzati, D., Campana, S., \& Ghisellini, G. 1999, MNRAS, 304, L31

Lazzati, D., Covino, S., \& Ghisellini, G. 2002a, MNRAS, 330, 583

Lazzati, D., Ramirez-Ruiz, E., \& Rees, M. J. 2002, ApJ, 572, L57

Li, Z., \& Chevalier, R. A. 1999, ApJ, 526, 716

Liedahl, D. A., Osterheld, A. L., \& Goldstein, W. H. 1995, ApJ, 438, L115

Meszaros, P., \& Rees, M. J. 1997, ApJ, 476, 232

Mewe, R., Gronenschild, E. H. B. M., \& van den Oord, G. H. J. 1985, A\&AS, 62, 197

Panaitescu, A., \& Kumar, P. 2002, ApJ, 571, 779

Piro, L., Garmire, G., Garcia, M., et al. 2000, Science, 290, 955

Piro, L., Frail, D. A., Gorosabel, J., et al. 2002, ApJ, 577, 680

Reeves, J. N., Watson, D., Osborne, J. P., et al. 2002, Nature, 416, 512 (R02)

Rybicki, G. B., \& Lightman, A. P. 1979, Radiative processes in astrophysics (New York: Wiley-Interscience), 1979

van Paradijs, J., Kouveliotou, C., \& Wijers, R. A. M. J. 2000, ARA\&A, 38, 379

Vietri, M., \& Stella, L. 1998, 507, L45

Vietri, M., Perola, C., Piro, L., \& Stella, L. 1999, MNRAS, 308, L29

Watson, D., Reeves, J. N., Osborne, J., et al. 2002, A\&A, 393, L1 